\title{
La bourgeoisie berlinoise et les premières œuvres impressionnistes de la Galerie nationale du temps de l’Empire : un mécénat « juif »?
}

Die Berliner Bourgeoisie und die ersten impressionistischen Werke der

Nationalgalerie im Deutschen Kaiserreich - Ein ,jüdisches“ Mäzenatentum? The Berlin Bourgeoisie and the First Impressionist Works of the National Gallery in the German Empire - A 'Jewish' Patronage?

Johanna Heinen

\section{OpenEdition}

Journals

Édition électronique

URL : https://journals.openedition.org/ceg/7070

DOI : 10.4000/ceg.7070

ISSN : 2605-8359

Éditeur

Presses Universitaires de Provence

Édition imprimée

Date de publication : 25 septembre 2019

Pagination : 75-91

ISBN : 979-10-320-0234-6

ISSN : 0751-4239

\section{Référence électronique}

Johanna Heinen, « La bourgeoisie berlinoise et les premières œuvres impressionnistes de la Galerie nationale du temps de l'Empire : un mécénat « juif »? », Cahiers d'Études Germaniques [En ligne], 77 | 2019, mis en ligne le 25 mars 2021, consulté le 15 juin 2021. URL : http://journals.openedition.org/ ceg/7070 ; DOI : https://doi.org/10.4000/ceg.7070 


\title{
La bourgeoisie berlinoise et les premières œuvres impressionnistes de la Galerie nationale du temps de l’Empire : un mécénat « juif »?
}

\author{
Johanna HEINEN \\ École des hautes études en sciences sociales, Paris / Freie Universität Berlin
}

II existe de nombreuses publications sur Hugo von Tschudi, I'ancien directeur de la Galerie nationale de Berlin, qui défia la doctrine artistique de l'empereur Guillaume II, en achetant dès 1896 des œuvres d'art avant-gardistes, provenant de surcroît de l'ennemie jurée : la France. Jusqu'à la fin de son mandat en 1909, qui s'acheva par une démission "forcée", Tschudi réussit à pourvoir le musée d'une impressionnante collection de chefs-d'œuvre, parmi lesquels des Courbet, Manet, Millet, Daubigny, Fantin-Latour, Monet, Renoir, Cézanne, Sisley, Pissarro, Vuillard, Bourdelle, Rodin et Maillol. La Galerie nationale joua ainsi un rôle de précurseur dans l'institutionnalisation de l'art moderne français, surtout de l'impressionnisme, en Allemagne, mais aussi en France. Mais qui étaient les mécènes prêts à soutenir ces achats audacieux et quelles étaient leurs motivations? De nombreuses publications sur l'art moderne à Berlin sous Guillaume II constatent une " surreprésentation juive » parmi les mécènes et collectionneurs allemands. Celle-ci est le plus souvent expliquée, d'une part, par des relations familiales et professionnelles étroites au sein de la communauté juive et, d'autre part, par des qualités supposées, telles qu'une grande ouverture d'esprit, voire un certain cosmopolitisme, qui aurait contribué à forger un modernisme prononcé ${ }^{1}$. S'y ajoutent d'autres thèses, notamment celle qui présente ces personnes comme faisant partie d'un groupe séparé et refermé sur lui-même ${ }^{2}$, ou bien encore la thèse qui affirme qu'au contraire, leur engagement constitue un affront politique

1. Notamment Stefan Pucks, « Von Manet zu Matisse. Die Sammler der französischen Moderne in Berlin », in Johann Georg Prinz von Hohenzollern, Peter-Klaus Schuster (dir.), Manet bis van Gogh. Hugo von Tschudi und der Kampf um die Moderne, München, Prestel, 1996, p. 389; Ines Sonder, " "Ein Ruhmesblatt für die Stifter". Mäzene der französischen Moderne für die Nationalgalerie um 1900 », in Anna-Dorothea Ludewig, Julius H. Schoeps, Ines Sonder (dir.), Aufbruch in die Moderne. Sammler, Mäzene und Kunsthändler in Berlin 1880-1933, Köln, DuMont 2012, p. 218; CellaMargaretha Girardet, Jüdische Mäzene für die Preußischen Museen zu Berlin. Eine Studie zum Mäzenatentum im Deutschen Kaiserreich und in der Weimarer Republik, Egelsbach/ Frankfurt a. M./ Washington, Hänsel-Hohenhausen, 1997, p. 29.

2. Veronica Grodzinski, French Impressionism and German Jewish patronage. The Reception of Modernism in Imperial Germany 1896-1914, PhD-dissertation, University College London, 2005, p. 269-271; id., « Collecting against the Grain », Jewish Quarterly 53 (2), 2006, p. 38-42. 
de la part d'une bourgeoisie bien établie et sûre d'elle-même dans le contexte du régime wilhelmien, et ce indépendamment de la religion ${ }^{3}$. Le fait que certains des mécènes aient été des Kaiserjuden, c'est-à-dire des Juifs proches de Guillaume II, et que d'autres aient été baptisés depuis plusieurs générations, invite cependant à questionner la validité de ces approches. D'autres publications sur l'histoire de l'art moderne en Allemagne à l'époque wilhelmienne constatent que les Juifs actifs dans le domaine étaient plus "allemands" que "juifs" et que même sans eux, l'art moderne se serait imposé ${ }^{4}$. Cependant, les trois quarts des mécènes qui ont financé les œuvres avant-gardistes françaises pour Hugo von Tschudi étaient également juifs.

Cet article présente un résumé des recherches antérieures menées par l'auteure ${ }^{5}$, qui s'est attachée à analyser les raisons ayant motivé le mécénat d'œuvres d'art moderne françaises offertes à la Galerie nationale entre 1896 et 1909, afin de déterminer si et dans quelle mesure l'origine juive des mécènes avait joué un rôle. Dans un souci de cohérence, la période analysée commence ici en 1882, année d'arrivée des premiers tableaux impressionnistes à Berlin, et se termine avec l'affaire du legs Tschudi, à la mort de ce dernier, en 1911, soit deux ans après son départ de la Galerie nationale pour la Pinacothèque de Munich.

\section{L'identité des mécènes}

On ne savait jusque-là que très peu de choses au sujet de certains des mécènes, sans doute parce que Hugo von Tschudi n'avait légué aucun journal de travail ni de mémoires personnels concernant ses activités et contacts parmi la bourgeoisie berlinoise. Cependant, on retrouve le nom, et souvent aussi la profession ainsi que l'adresse de chaque mécène, sur les demandes d'autorisation que Tschudi envoyait à l'empereur avant qu'une œuvre offerte ou une nouvelle acquisition ne vienne rejoindre la collection de la Galerie nationale de Berlin. II s'agissait donc dans un premier temps de retrouver l'identité des quarante-cinq mécènes d'art moderne étrangers actifs à l'époque où Tschudi était directeur de la Galerie nationale. La source principale de ces recherches fut le registre historique de la préfecture de police de Berlin, conservé au Landesarchiv ${ }^{6}$, les archives du Land de Berlin. Elles abritent un dossier personnel pour presque chacun des

3. Eberhard Roters, "Die Nationalgalerie und ihre Stifter », in Günter Braun, Waltraud Braun (dir.), Mäzenatentum in Berlin: Bürgersinn und kulturelle Kompetenz unter sich verändernden Bedingungen, Berlin/ New York, De Gruyter, 1993, p. 86-87.

4. Notamment Peter Paret, «Bemerkungen zu dem Thema: Jüdische Kunstsammler, Stifter und Kunsthändler », in Ekkehard Mai, Peter Paret, Ingrid Severin (dir.), Sammler, Stifter und Museen: Kunstförderung in Deutschland im 19. und 20. Jahrhundert, Köln/ Weimar/ Wien, Böhlau, 1993, p. 173-175; Peter Gay, Freud, Juden und andere Deutsche. Herren und Opfer in der modernen Kultur, München, DTV, 1989, p. 115.

5. Johanna Heinen, Ein „jüdisches" Mäzenatentum für moderne französische Kunst? Das Fallbeispiel der Nationalgalerie im Berlin der wilhelminischen Ära (1882-1911), Frankfurt a. M., Peter Lang, 2016.

6. Landesarchiv de Berlin (LAB), A Pr.Br.Rep. 030. 
mécènes. Ces documents recueillaient des informations détaillées sur chaque membre de l'élite économique avant que ne lui soit décerné éventuellement un ordre ou une distinction : parcours professionnel, situation financière, activités politiques, appartenance religieuse, style de vie, contacts personnels. Des recherches effectuées dans les registres de dix archives complémentaires ${ }^{7}$, des informations obtenues par les descendants des mécènes, par d'autres chercheurs et universitaires ainsi que dans des journaux de l'époque et de nombreuses publications interdisciplinaires sont ensuite venues enrichir cette documentation.

L'une des découvertes les plus incongrues fut de constater que les chercheurs avaient dans leurs publications jusqu'alors "marié" à tort la mécène viennoise Henriette Mankiewicz au banquier berlinois Paul Mankiewitz, sans doute en raison de l'homophonie de leurs noms de famille. En outre, on ignorait jusque-là que trois associés de Robert Warschauer \& Co. étaient des mécènes de la Galerie nationale. De plus, Karl Steinbart, un collectionneur d'art moderne français et chef du personnel chez Mendelssohn \& Co., avait été considéré comme juif dans toutes les publications en histoire de l'art, alors qu'il était protestant et n'avait aucune ascendance juive.

Les vingt-huit personnes composant le panel des analyses principales remplissaient toutes les mêmes critères de sélection : elles eurent une adresse berlinoise entre 1882 et 1911 et furent mécènes d'au moins une œuvre d'art française appartenant à un style non encore reconnu académiquement au sein de l'Empire wilhelmien.

Tous ces donateurs étaient millionnaires (tableau 1), deux tiers d'entre eux étaient banquiers, d'autres étaient grossistes en charbon ou dans l'industrie sidérurgique (tableau 2). Seuls trois mécènes exerçaient des professions intellectuelles : le peintre Max Liebermann ainsi que deux professeurs de l'actuelle université Humboldt de Berlin. Eux aussi étaient issus de familles respectées de banquiers et d'industriels. Vingt des vingt-huit mécènes étaient d'origine juive (tableau 3). Cependant, six d'entre eux n'avaient pas grandi dans la religion juive puisque leurs parents étaient déjà baptisés à leur naissance. C'était aussi le cas pour tous les membres des grandes familles de banquiers Mendelssohn \& Co. et Robert Warschauer \& Co. Deux autres mécènes se convertirent au protestantisme à l'âge adulte. Par conséquent, seuls douze d'entre eux restèrent membres de la communauté juive jusqu'à la fin de leur vie, ce qui représente moins de la moitié du panel. Parmi les mécènes se trouvait aussi une catholique.

Au regard du pourcentage de la population juive à Berlin - environ $4 \%$ de la population totale de la ville - voire au niveau national - environ $1 \%-$, on constate une immense « surreprésentation juive » dans les domaines de la collection et du commerce d'art, ainsi que dans la donation d'œuvres d'art moderne français à

7. Archives complémentaires consultées : Archiv der Alten Nationalgalerie im Zentralarchiv, Staatliche Museen zu Berlin - Preußischer Kulturbesitz; Geheimes Staatsarchiv Preußischer Kulturbesitz, Berlin; Archiv des Leo Baeck Instituts im Jüdischen Museum Berlin; Stiftung Neue Synagoge Berlin - Centrum Judaicum; Evangelisches Zentralarchiv, Berlin; Wiener Stadt- und Landesarchiv; Stadtarchiv Magdeburg ; Landesarchiv Nordrhein-Westfalen; Archives de Paris. 
la Galerie nationale. Pour définir la catégorie de "Juif », la recherche s'appuyait souvent jusque-là sur la notion d'« ethnicité » telle qu'on la trouve chez Werner E. Mosse et qui englobe aussi les personnes baptisées mais ayant une ascendance juive, même lointaine. Selon Mosse, ce " groupe ethnique " avait pour base commune des caractéristiques telles que le mariage au sein de la communauté, des réseaux familiaux, des us et coutumes communs ainsi que la préservation de noms typiques; la religion n'y figurait donc que comme une caractéristique parmi d'autres ${ }^{8}$.

Il est toutefois important de mettre le nombre de mécènes juifs en rapport avec leur représentation dans le milieu social concerné, et non pas tant avec le pourcentage de la population nationale. D'après une étude de Dolores Augustine $^{9}$, trois quarts des millionnaires issus de l'élite économique berlinoise étaient juifs dans le sens de cette ethnicité. Cela correspond au pourcentage de Juifs parmi les mécènes berlinois d'art moderne français. Ce fait réfute ainsi la thèse de la surreprésentation, étant donné que la richesse était une condition sine qua non pour être mécène. Reste la question de savoir pourquoi la majorité des Juifs berlinois étaient millionnaires. Les analyses de Stefi Jersch-Wenzel ${ }^{10}$ ont permis de démontrer que cette richesse s'explique par des raisons historiques. En mars 1812, les Juifs installés en Prusse obtinrent finalement le droit de devenir des citoyens prussiens. Jusqu'à cette date, seuls des Juifs extrêmement aisés recevaient la permission de s'installer durablement dans la ville de Berlin. Cette autorisation était soumise à des conditions et obligations, notamment au paiement de taxes très élevées. De plus, dans la ville de Berlin, le régime prussien accordait des privilèges aux industriels juifs dans les domaines qui lui étaient nécessaires, comme le textile. La restriction de l'accès à certains métiers mena aussi, c'est un fait connu, à une concentration de la population active juive dans le domaine bancaire. Au regard des dates de naissance des mécènes, il est probable que leurs parents ou grands-parents avaient encore été affectés par ces règlementations. À partir de 1888, Guillaume II, le dernier empereur allemand, encouragea fortement l'ouverture de son pays sur le marché mondial et sa modernisation industrielle, notamment en ce qui concerne Berlin. Sous son règne, les banques commencèrent à jouer un rôle majeur dans le pays, tout particulièrement dans la capitale. Ainsi, une large partie des grandes banques allemandes vinrent s'y installer.

Seuls neuf des vingt-huit mécènes étaient berlinois de naissance, et trois autres avaient passé leur enfance dans la ville. Le panel comporte par ailleurs plusieurs directeurs de grandes banques. Malgré l'assimilation formelle des Juifs en tant que citoyens de l'Empire allemand, ils demeurèrent souvent actifs dans les mêmes secteurs professionnels que leurs ancêtres - sans doute motivés par

8. Werner E. Mosse, Jews in the German Economy. The German-Jewish Economic Élite 1820-1935, Oxford, Clarendon Press, 1987, p. 2.

9. Dolores L. Augustine, « Die jüdische Wirtschaftselite im wilhelminischen Berlin: Ein jüdisches Patriziat? », in Reinhard Rürup (dir.), Jüdische Geschichte in Berlin. Essays und Studien, Berlin, Hentrich, 1995, p. 101-116.

10. Stefi Jersch-Wenzel, « Der Neubeginn jüdischen Lebens in Berlin seit 1671: Die ersten Generationen », in Rürup, Jüdische Geschichte in Berlin, p. 13-24. 
la conjoncture favorable à leurs entreprises et par les distinctions reçues sous Guillaume II.

Cette première étape d'analyse a donc permis, non seulement de vérifier les identités des mécènes constituant le panel, mais aussi d'étoffer la documentation les concernant. En outre, elle a conduit à réfuter la thèse de la « surreprésentation juive » parmi les mécènes d'art moderne français de la Galerie nationale. Cependant, il s'agissait aussi de comprendre leurs motivations : pourquoi, en effet, contribuer à financer la constitution d'une collection d'œuvres d'art issues d'un courant encore si controversé politiquement à l'époque?

\section{Cadre, mécanismes et signification du mécénat à Berlin à l'ère wilhelmienne}

En évaluant la part proportionnelle des dons, on se rend compte que l'importance du mécénat d'art moderne français pour la Galerie nationale avait été largement surestimé jusque-là : deux tiers des mécènes ne furent actifs qu'une seule fois pour ces œuvres et seize des montants versés s'élevaient au maximum à 5000 marks, une somme dérisoire au regard des dons effectués pour d'autres courants artistiques et projets culturels. Les sommes versées par les mécènes juifs n'étaient pas plus élevées en moyenne que celles des autres mécènes du panel. Seule exception : le banquier juif Carl Hagen, un mécène quasiment inconnu, qui finança à lui seul près d'un tiers de l'ensemble des achats d'œuvres d'art moderne français pour la collection de la Galerie nationale.

Parmi les sept mécènes à avoir contribué à financer l'achat d'art moderne français avec des sommes supérieures à 20000 marks, on retrouve d'une part Carl Hagen et Eduard Arnhold, tous deux de confession juive, d'autre part Robert Mendelssohn, chrétien d'ascendance juive, ainsi que les chrétiens sans ascendance juive Felix Koenigs, Karl Steinbart, Paul Merling et Henckel von Donnersmarck. Ce dernier était issu de la noblesse et proche de Guillaume II.

En outre, il ne s'agissait pas de donations d'œuvres provenant de collections privées, comme on l'avait supposé jusqu'alors, mais de dons d'argent, sauf dans deux cas de legs après décès d'un mécène (Felicie Bernstein par exemple). Seuls onze des vingt-huit mécènes possédaient eux-mêmes des œuvres d'art moderne dans leurs collections privées, et seuls huit au moins une œuvre impressionniste. L'ensemble de ces résultats permet de réfuter la thèse selon laquelle il existait une homogénéité entre les donateurs et les collectionneurs d'art moderne français. Dans le panel, il manque en effet les noms des grands collectionneurs berlinois d'art moderne de l'époque.

Parmi tous les mécènes juifs, les Bernstein étaient en outre les seuls à entretenir des relations familiales et commerciales avec l'avant-garde française, un aspect crucial qui explique leur intérêt pour ce courant artistique. Ils achetèrent les premières œuvres impressionnistes en 1882. Mais ce n'est qu'à partir des années 1890 que d'autres membres de la bourgeoisie berlinoise commencèrent 
également à les collectionner. À cette époque, les œuvres avaient déjà acquis une grande valeur sur le marché artistique. Les collectionneurs ne prenaient donc pas de risques.

La présence d'un libéralisme et d'un modernisme artistiques très prononcés chez les mécènes est à relativiser : aucun d'entre eux ne fit appel à un architecte moderne, comme par exemple Van de Velde, pour faire aménager son intérieur dans le style Art Nouveau et ils n'apportèrent pas leur soutien à de jeunes artistes français méconnus afin de les aider à percer. On ne trouve ainsi que peu d'œuvres néo-impressionnistes, fauvistes, cubistes et expressionnistes dans leurs collections privées.

Sur quels mécanismes, sur quel habitus, reposaient alors le mécénat et la constitution de collections d'art privées au sein de la bourgeoisie berlinoise? Pour cerner cette question, I'analyse de Pierre Bourdieu ${ }^{11}$ sur les différentes formes de capital ${ }^{12}$ et sur leur «transformabilité » s'est avérée utile. Une analyse géographique des lieux de résidence et d'affaires des mécènes, basée sur la création préalable de cartes historiques ${ }^{13}$, a permis de constater que les premières collections d'art de l'élite économique apparurent seulement à partir de 1885. Et ce, à la suite de déménagements, en règle générale de l'appartement situé au sein de l'entreprise familiale dans le quartier d'affaires de Berlin Mitte, qui était alors le centre de la ville, pour une villa, souvent située dans le quartier prestigieux de Tiergarten.

Contrairement à ce que l'on avait supposé jusque-là, on ne trouve à cette époque à Tiergarten que des collections et des donations d'œuvres s'inscrivant dans des courants artistiques reconnus. C'est Wilhelm von Bode, lui-même directeur général des musées royaux de Berlin à partir de 1905, qui mit en place cet habitus de prestigieuse collection d'art dans les nouvelles villas des mécènes, comprenant également de formidables décorations d'intérieur, souvent dans le style Renaissance ou Rococo.

En outre, il engagea au sein de la bourgeoisie berlinoise une transition du statut de collectionneur à celui de mécène. II sut se rendre indispensable en aménageant les villas et en acquérant des objets d'art historiques pour ses clients. Il organisa aussi des expositions de collections privées ouvertes au public et rédigea dans ce cadre d'impressionnants catalogues ${ }^{14}$. En échange, il demandait ou du moins espérait le soutien des mécènes pour les musées royaux berlinois. II sut habilement mettre en contact les collectionneurs entre eux, en les faisant entrer dans les clubs élitistes des musées berlinois, dont les cotisations étaient très élevées. Ce rendez-vous devint presque obligatoire pour les membres de la bourgeoisie berlinoise qui souhaitaient maintenir leur statut social au sein de la communauté commerçante. D'autres membres espéraient quant à eux améliorer

11. Notamment Pierre Bourdieu, Les Règles de l'art. Genèse et structure du champ littéraire, $2^{e}$ édition, Paris, Seuil, 1998.

12. Culturel, social, symbolique et économique.

13. Heinen, Ein „jüdisches“" Mäzenatentum, p. 363-372.

14. Wilhelm von Bode, Mein Leben, vols. 1 et 2, Berlin, Verlag Hermann Reckendorf, 1930. II publia également des livres sur les collections privées de James Simon, Oscar Huldschinsky, Alfred Beit et Marcus Kappel. 
par là leur statut social de manière significative. Cet habitus faisait miroiter à la bourgeoisie berlinoise l'accès à un capital social et culturel, transférable par la suite en capital économique grâce aux nouveaux contacts et au surcroît de prestige.

Dans ces milieux, une collection d'art moderne permettait de démontrer une certaine individualité, mais seulement dans la mesure où le statut social et la position acquise au sein d'un groupe défini le permettaient. Le libéralisme artistique ne se vivait donc que jusqu'au seuil de tolérance défini par le cercle social de la bourgeoisie berlinoise, indépendamment de la religion. Ainsi même les Bernstein étaient-ils plutôt "vieille école" en ce qui concerne le code de conduite en société et l'aménagement de leurs appartements ${ }^{15}$.

Le mécénat d'art moderne français pour la Galerie nationale semble dans la plupart des cas bien modeste, comparé aux autres dons de chaque mécène. Cela s'explique par le fait que, par rapport à Wilhelm von Bode, Hugo von Tschudi n'était pas en mesure de rendre autant de services aux mécènes en contrepartie de leurs dons : deux tiers d'entre eux ne s'intéressaient pas personnellement à l'art moderne français. Et ceux qui étaient intéressés n'avaient pas besoin de Tschudi pour acquérir des œuvres. Lorsque Tschudi prit son poste à la Galerie nationale, d'autres experts et médiateurs étaient déjà actifs dans le domaine, comme par exemple Max Liebermann. Deux ans plus tard, en 1898, apparut en outre le Kunstsalon Bruno \& Paul Cassirer, un salon artistique d'art moderne. Les mécènes qui souhaitaient présenter à leurs invités une collection d'art moderne privée exceptionnelle, souvent dans une salle séparée, n'avaient sans doute que peu d'intérêt à voir ces mêmes œuvres déjà exposées et reconnues à la Galerie nationale.

En revanche, on pourrait expliquer les importantes sommes d'argent versées par quelques mécènes à la Galerie nationale par une volonté d'effectuer un geste patriotique pour renforcer la compétitivité des musées de leur ville et patrie d'adoption au niveau international. Cette thèse semble être confortée par les faits qui eurent lieu suite au legs Tschudi, tels qu'ils sont rapportés par d'autres auteurs $^{16}$. Lorsque Hugo von Tschudi décéda en 1911, il était directeur de la Pinacothèque de Munich depuis sa démission de Berlin en 1909. Avant de quitter la Galerie nationale, Tschudi avait acheté de nombreuses œuvres d'art grâce aux dons et prêts de membres de la bourgeoisie berlinoise. Parmi ces œuvres se trouvaient notamment des peintres encore plus avant-gardistes que ceux exposés jusque-là à la Galerie nationale, tels Paul Gauguin, Vincent van Gogh et Henri Matisse ${ }^{17}$. À la mort de Tschudi, les Berlinois Eduard Arnhold et Robert Mendelssohn renoncèrent aux remboursements de leurs crédits accordés

15. Max Liebermann, Die Phantasie in der Malerei: Schriften und Reden, Frankfurt a. M., Fischer Verlag, 1978, p. 99.

16. Christian Lenz, "Heinz Braune und die Tschudi-Spende », in Prinz von Hohenzollern, Schuster (dir.), Manet bis van Gogh, p. 432-437; Michael Dorrmann, Eduard Arnhold (1849-1925). Eine biografische Studie zu Unternehmer- und Mäzenatentum im Deutschen Kaiserreich, Berlin, Akademie Verlag, 2002, p. 158.

17. Il s'agit ici du tableau Nature morte aux géraniums, la première œuvre du peintre à être exposée dans un musée européen. 
à l'ancien directeur de la Galerie nationale afin de soulager sa veuve, mais ils s'engagèrent dans un vif débat avec les autres donateurs pour que ces œuvres avant-gardistes soient retournées à la Galerie nationale de Berlin, et non offertes à Munich ${ }^{18}$.

Ce fort attachement patriotique à la ville de Berlin se retrouve aussi dans certains choix personnels parmi les mécènes du panel. Ainsi, sur les vingt-deux personnes dont on connaît avec certitude le lieu de sépulture, dix-sept ont choisi d'être inhumées dans leur patrie d'adoption, Berlin ${ }^{19}$, bien que seuls neuf y soient nés. Seuls trois mécènes furent enterrés dans leur ville d'origine, aucun d'entre eux n'était d'ascendance ou de religion juive.

Même si les mécènes étaient très attachés à leur ville, pourquoi finançaientils des œuvres d'art moderne français dont le style et le pays de provenance ne correspondaient ni à la doctrine artistique ni à la politique suivie par Guillaume II?

\section{Affront politique et antisémitisme? Les liens entre les mécènes et le régime prussien}

Tschudi n'ayant laissé presque aucun document sur la provenance et les montants des dons versés par les mécènes, il est difficile de reconstituer le cadre dans lequel s'effectuait ce mécénat. Les dons d'une partie des mécènes pourraient être interprétés comme un geste de bienvenue à l'arrivée du nouveau directeur ou, de manière plus générale, comme le souhait d'enrichir la collection de la Galerie nationale. Ainsi, même Guillaume II effectua des dons issus de sa fortune personnelle ${ }^{20}$ pour financer des achats au cours de la première année de Tschudi à la Galerie nationale. L'empereur voyait dans les musées un symbole de la suprématie de son pays. Pour lui, la Galerie nationale jouait un rôle majeur dans l'éducation culturelle de la population. Avant d'arriver à la Galerie nationale, Hugo von Tschudi était un employé très assidu, formé aux musées royaux de Berlin sous la direction de Wilhelm von Bode. En le nommant à la tête du musée berlinois, on ne pensait pas que le nouveau directeur s'engagerait avec autant de ferveur en faveur de l'art moderne français et les courants artistiques non reconnus académiquement. Après de nombreuses années en tant qu'assistant de Wilhelm von Bode, Tschudi vit dans son nouveau poste la possibilité de créer et de réaliser pour la première fois de nouveaux projets pour les collections du musée et de transmettre une nouvelle doctrine artistique au public. À sa demande, Max Liebermann l'initia au milieu artistique parisien avant-gardiste. Et pourtant, même

18. Conformément aux derniers vœux de Tschudi, au moins 33 œuvres rejoignirent finalement la collection de la Pinacothèque de Munich.

19. Y compris les faubourgs avoisinants qui font aujourd'hui partie de la ville.

20. À l'époque, il était interdit d'utiliser des fonds publics pour acquérir des œuvres étrangères, même celles s'inscrivant dans des styles reconnus académiquement et appréciés par Guillaume II. Seul le financement par le biais de dons privés était possible. 
Max Liebermann avait ses réserves quant aux nouvelles idées enthousiastes de Hugo von Tschudi pour la Galerie nationale.

Peut-on considérer le soutien financier des mécènes comme un affront politique? Aucun nom de mécène ne figurait, comme cela se fait de nos jours, sur les plaquettes accompagnant chaque objet d'art. Et seul Paul Merling souhaitait expressément être mentionné dans les catalogues annuels de la Galerie nationale. En outre, on ne retrouve que très peu de notes de ces versements dans les dossiers de police, et jamais dans un sens négatif.

En ce qui concerne les liens des vingt-huit mécènes avec le gouvernement prussien, il existe encore plusieurs exemples attestant que leur engagement auprès de la Galerie nationale n'affecta en aucun cas les bonnes relations qu'ils entretenaient avec lui. Au moins huit mécènes, parmi lesquels figuraient également des Juifs, entretenaient des contacts réguliers avec l'empereur. On lui prêtait cependant un antisémitisme prononcé et à part quelques exceptions, les citoyens d'ascendance juive de cette époque ne pouvaient pas aspirer à une carrière politique ou militaire. Même si les relations qu'entretenait Guillaume II avec ces membres de l'élite de la bourgeoisie berlinoise n'avaient aucun caractère amical, elles reposaient toutefois sur un respect mutuel. Seul le mécène Paul Merling, qui n'était pas juif et qui n'entretenait pas de contacts réguliers avec Guillaume II, rendit en 1896 la nationalité prussienne pour devenir citoyen du Duché de Saxe-Cobourg et Gotha. Néanmoins, il reçut plusieurs décorations de la ville de Berlin, notamment une première en 1892, pour honorer son engagement en tant que mécène d'art moderne au profit de la Galerie nationale ${ }^{21}$.

Eduard Arnhold était non seulement l'un des mécènes les plus importants de la Galerie nationale, mais également lui-même collectionneur renommé d'art moderne français. Et pourtant, il fut élu à la Preußische Herrenhaus en 1913; il devint alors le premier Juif non converti à intégrer cette assemblée en quarante ans.

Quant à Carl Hagen, qui finança à lui seul presque un tiers de l'ensemble des dons versés à la Galerie nationale pour sa collection d'œuvres d'art moderne françaises, il recevait régulièrement des hauts fonctionnaires dans le cadre de ses activités économiques très prospères et il siégea au conseil d'administration de treize banques et entreprises industrielles. Pendant la Première Guerre mondiale, il mit sa résidence d'été de Potsdam à disposition de l'armée afin qu'y soit installé un hôpital militaire. En outre, son gendre Ernst David fut l'un des rares officiers d'origine juive reconnus. Eduard Arnhold et Carl Hagen restèrent toute leur vie membres de la communauté juive. Tout comme d'autres mécènes de la bourgeoisie berlinoise, ils semblent n'avoir été ni ciblés ni influencés de manière notable par l'antisémitisme.

Les membres du panel étudié ici soutenaient avec beaucoup d'engagement et des dons bien plus élevés que ceux versés à la Galerie nationale, des projets culturels, économiques ou sociaux, conçus, dirigés ou du moins fortement

21. LAB, A Pr.Br.Rep. 030, Nr. 11771. 
appréciés par le régime prussien. De manière générale, pour la bourgeoisie berlinoise, ces nombreux mécénats étaient une des conditions préalables à l'obtention de titres et distinctions décernés par l'Empire. En outre, le gouvernement ne permettait pas seulement à ces commerçants berlinois d'élever ainsi leur statut social, il leur accordait également son soutien et les autorisations nécessaires pour favoriser la croissance économique de leurs entreprises, et ce, également à l'étranger. En échange, Guillaume II profitait de la prospérité de la bourgeoisie qui lui accordait à son tour les emprunts nécessaires pour atteindre ses objectifs économiques et militaires.

Par ailleurs, les critères à remplir pour obtenir ces distinctions officielles se retrouvaient également dans les principes du code de l'honneur des marchands berlinois. Fait remarquable, ils disposaient d'un droit de codécision non négligeable lors de l'attribution des distinctions. L'Ältestenkollegium, comité de direction de l'association des marchands berlinois le plus influent de la ville, avec à sa tête une personne d'ascendance juive, renseignait régulièrement le gouvernement sur les performances économiques et la conduite morale de ses nombreux membres ${ }^{22}$. Le mécénat, même en ce qui concerne la majorité des œuvres d'art moderne français, faisait donc partie d'un double système d'aides et de récompenses entre les commerçants de la capitale et le gouvernement.

\section{Conclusion et perspectives}

Les résultats de notre analyse ont permis de réfuter les modèles prédominants d'explication des motivations du mécénat d'art moderne avant-gardiste français. Notre étude a pu d'une part démontrer qu'on ne saurait parler de " surreprésentation juive » parmi les mécènes d'art moderne français pour la Galerie nationale à l'époque de Guillaume II, car leur part correspond bien au pourcentage que l'on retrouve dans la bourgeoisie berlinoise, seul groupe véritablement actif dans le domaine. Les collectionneurs et mécènes d'art moderne à Berlin ne constituaient pas un groupe entièrement homogène quant à leurs préférences en matière d'art.

Cette étude interdisciplinaire a permis d'autre part de réfuter en grande partie la thèse d'une éventuelle influence de l'origine juive des mécènes. Ces derniers n'étaient pas en butte à l'antisémitisme au sein des structures de l'élite économique; le mécénat n'était donc pas non plus une tentative pour compenser un déficit, ni une façon de se démarquer symboliquement, du fait d'attitudes antisémites, d'un entourage non-juif. Nous avons ainsi également pu invalider la représentation du Juif comme prototype du cosmopolite moderne, et réfuter - à l'exception des Bernstein - la supposition selon laquelle les réseaux juifs transnationaux auraient exercé une influence décisive sur l'intérêt des mécènes juifs pour l'art moderne français. Le mécénat juif, mais aussi la promotion de l'art moderne français, sont

22. Les dossiers de chaque membre de la bourgeoisie berlinoise se trouvent au Landesarchiv de Berlin, cote A Pr.Br.Rep. 030. 
plutôt à placer dans la logique du champ social des marchands berlinois. L'art moderne leur permettait d'exprimer une forme d'individualisme dans leurs propres rangs. Les collectionneurs parmi nos mécènes respectaient cependant le seuil de tolérance accordé par leur entourage social, s'en tenant à l'art ancien pour l'aménagement de leurs propres résidences et investissant avec l'art moderne dans un capital sûr. Leur modernisme et leur libéralisme avaient donc des limites claires.

Par conséquent, le mécénat ne peut être interprété que chez peu de nos mécènes comme une véritable volonté d'aider l'art moderne français à être reconnu officiellement en Allemagne ou à un niveau international. Même si leur mécénat a eu une grande influence en ce sens, il ne représentait finalement pour la plupart d'entre eux qu'une activité mineure de soutien parmi tant d'autres, qui, de plus, existait avant que les conflits entre Hugo von Tschudi et l'empereur ne fissent scandale. Le fait que certains des mécènes aient perpétué leurs activités de mécénat au profit de la Galerie nationale malgré la politisation croissante des œuvres d'art s'explique notamment par leur patriotisme local et la volonté de rendre les musées de leur nouvelle "patrie » plus compétitifs à l'échelle internationale. Les résultats de cette étude montrent que les personnes d'origine juive représentaient une partie intégrante, fondamentale et active de l'élite économique berlinoise, et qu'elles ne sauraient de ce fait être traitées comme un groupe à part dans les travaux de recherche s'interrogeant sur la promotion culturelle et artistique. Une lecture dans le sens d'une préhistoire du génocide n'est en aucun cas adaptée. Notons cependant qu'en histoire de l'art, on a encore tendance à parler de personne "juive » simplement parce que celle-ci s'est engagée dans le domaine de l'art moderne étranger ou parce qu'elle travaillait dans une entreprise portant un nom juif. On ne vérifie pas toujours quelle était sa confession à l'époque ou quelle avait été celle de ses ancêtres. Karl Steinbart, chef du personnel chez Mendelssohn \& Co., collectionneur d'art moderne et l'un des mécènes de notre étude, a été jusqu'à ce jour toujours rattaché à la communauté juive, alors qu'il alors qu'il n'avait aucune ascendance juive. En outre, la question de savoir si la religion pourrait avoir eu une influence sur le goût ou l'engagement pour les arts modernes à l'époque, une thèse qui ne pourrait être vérifiée que par des recherches pluridisciplinaires, reste souvent ignorée. Cet amalgame récurrent n'est pas sans rappeler, si bonnes que soient les intentions de l'auteur, les catégorisations d'un certain discours antisémite.

La ville de Berlin, les relations de proximité avec l'empereur et avec les hauts responsables économiques de l'administration prussienne, ainsi que l'évolution particulière de la situation juridique de la communauté juive dans la capitale et I'habitus du mécénat et des collections d'art parmi la bourgeoisie berlinoise ont été des facteurs déterminants dans la définition des résultats de cette étude. On peut donc supposer qu'une analyse des motivations du mécénat et notamment de l'influence de l'origine juive sur celui-ci dans d'autres villes comme Francfort, Hambourg ou Munich, offrant chacune un cadre tout à fait différent, livrerait certainement d'autres conclusions. II serait de même intéressant d'étendre les recherches à d'autres catégories de médiateurs d'art, tels que les collectionneurs, 
les marchands d'art, les intermédiaires, etc., afin d'analyser les motivations de ces derniers dans leur contexte local respectif et de montrer l'interaction et les réseaux transnationaux entre les différents acteurs et groupes de médiateurs. Une analyse de la réception de l'art avant-gardiste français et des influences possibles d'une origine juive sur les motivations des personnes ayant promu ce courant, en s'attardant sur chaque ville de l'Empire allemand et de la République de Weimar n'a pas encore été effectuée. Une analyse qui se pencherait sur l'ensemble de l'espace germanophone (avec l'Autriche et la Suisse), voire sur l'Europe entière, constitue un desideratum qui devrait devenir un objectif de la recherche dans ce domaine. C'est seulement ainsi que l'on pourra éviter que des stéréotypes à caractère antisémite sous-jacent, comme celui du Juif comme prototype du cosmopolite moderne, ne soient perpétuellement repris sans être questionnés, et que la représentation du Juif errant continue à être répétée dans l'histoire de l'art. 
Tableau 1 - La fortune personnelle des 28 mécènes, 1910-191123

\begin{tabular}{|c|c|c|}
\hline $\begin{array}{l}\text { (A) au moins } 50 \text { millions } \\
\text { de Marks (MM) }\end{array}$ & 1 & $\begin{array}{l}\text { Guido Henckel von Donnersmarck (254 MM), } \\
\text { Guillaume II (144 MM) }\end{array}$ \\
\hline (B) 40-49 MM & $\begin{array}{l}2 \\
3 \\
4\end{array}$ & $\begin{array}{l}\text { Fritz von Friedländer-Fuld (46 MM) } \\
\text { Ernst von Mendelssohn-Bartholdy (43 MM) } \\
\text { Eduard Arnhold ( } 40 \mathrm{MM})\end{array}$ \\
\hline (C) 30-39 MM & & $\begin{array}{l}\text { James Simon ( } 35 \mathrm{MM} \text { ) } \\
\text { Georg von Bleichröder (environ } 30 \mathrm{MM} \text { ) }\end{array}$ \\
\hline (D) 20-29 MM & & $\begin{array}{l}\text { Oscar Huldschinsky (27 MM) } \\
\text { Robert von Mendelssohn ( } 25 \mathrm{MM}) \\
\text { Franz von Mendelssohn jun. (22 MM) }\end{array}$ \\
\hline (E) 10-19 MM & $\begin{array}{l}10 \\
11\end{array}$ & $\begin{array}{l}\text { Julius Bleichröder (19 MM) } \\
\text { Isidor Loewe, Robert Warschauer junior, Eduard Veit } \\
\text { et Felix Koenigs (mécène : son épouse Elise Koenigs) } \\
\text { ( } 14 \mathrm{MM}) \\
\text { Max Steinthal ( } 13 \mathrm{MM}) \\
\text { Hugo Oppenheim (11 MM) }\end{array}$ \\
\hline (F) 1-9 MM & $\begin{array}{l}14 \\
15 \\
16 \\
17 \\
18 \\
19\end{array}$ & $\begin{array}{l}\text { Carl Hagen (7 MM) } \\
\text { Marcus Kappel (mécène : Mathilde Kappel) (8 MM) } \\
\text { Georg von Siemens et Max Liebermann ( } 6 \mathrm{MM}) \\
\text { Karl von der Heydt ( } 5 \mathrm{MM}) \\
\text { Paul von Merling ( } 4 \mathrm{MM}) \\
\text { Karl Steinbart, Karl Wesendonk, Wilhelm Staudt et Oscar } \\
\text { Hainauer ( } 2 \mathrm{MM}) \\
\text { Julius Kaufmann et Carl Bernstein (mécène : Felicie } \\
\text { Bernstein) (1 MM) }\end{array}$ \\
\hline
\end{tabular}

23. Johanna Heinen, Ein "jüdisches" Mäzenatentum, p. 58. En complément d'information, on pourra y trouver quatorze tableaux, cinq cartes et les biographies détaillées de quarante-cinq mécènes d'œuvres d'art moderne étrangères pour la Galerie nationale entre 1896 et 1909. 
Tableau 2 - Entreprises, secteurs d'activité et positions des mécènes ${ }^{24}$

\begin{tabular}{|c|c|c|c|}
\hline \multicolumn{2}{|c|}{ Nom, prénom } & \multirow{2}{*}{$\begin{array}{l}\text { Nom de l'entreprise } \\
\text { Secteur }\end{array}$} & \multirow{2}{*}{$\begin{array}{l}\text { Position } \\
\text { Associé et directeur }\end{array}$} \\
\hline & Arnhold, Eduard & & \\
\hline & $\begin{array}{l}\text { Bernstein, Felicie } \\
\text { et Carl }\end{array}$ & $\begin{array}{l}\text { Université Friedrich Wilhelms } \\
\text { Enseignement et sciences }\end{array}$ & Titulaire d'une chaire \\
\hline & $\begin{array}{l}\text { Bleichröder, Georg } \\
\text { Dr. junior }\end{array}$ & $\begin{array}{l}\text { S. Bleichröder } \\
\text { Banques et finances }\end{array}$ & Associé \\
\hline & Bleichröder, Julius & $\begin{array}{l}\text { Julius Bleichröder \& Co. } \\
\text { Banques et finances }\end{array}$ & Propriétaire \\
\hline & $\begin{array}{l}\text { Friedländer-Fuld, } \\
\text { Fritz }\end{array}$ & $\begin{array}{l}\text { Emmanuel Friedländer \& Co. } \\
\text { Commerce de gros de charbon }\end{array}$ & Propriétaire \\
\hline & $\begin{array}{l}\text { Hagen (jusqu'en } \\
1905 \text { : Levy), Carl }\end{array}$ & $\begin{array}{l}\text { Wiener, Levy \& Co., } \\
\text { Banques et finances }\end{array}$ & Propriétaire \\
\hline & $\begin{array}{l}\text { Hainauer, } \\
\text { Julie et Oscar }\end{array}$ & $\begin{array}{l}\text { S. Bleichröder u. Rothschild, } \\
\text { puis Oscar Hainauer } \\
\text { Système bancaire et finances }\end{array}$ & Employé, puis propriétaire \\
\hline & $\begin{array}{l}\text { Henckel von } \\
\text { Donnersmarck, } \\
\text { Guido }\end{array}$ & $\begin{array}{l}\text { Fürst von Donnermarck'schen } \\
\text { Bergwerks- und Hüttenbetriebe } \\
\text { Industrie et commerce (charbon, } \\
\text { zinc, soie, ...) }\end{array}$ & Propriétaire \\
\hline & von der Heydt, Karl & $\begin{array}{l}\text { Von der Heydt \& Co. } \\
\text { Banques et finances }\end{array}$ & Propriétaire \\
\hline & Huldschinsky, Oscar & $\begin{array}{l}\text { S. Huldschinsky \& Söhne } \\
\text { Industrie et commerce } \\
\text { (acier et sidérurgie) }\end{array}$ & $\begin{array}{l}\text { Co-fondateur, associé et } \\
\text { propriétaire des usines }\end{array}$ \\
\hline & $\begin{array}{l}\text { Kappel, Mathilde } \\
\text { et Marcus }\end{array}$ & $\begin{array}{l}\text { Is. Kappel } \\
\text { Banques et finances / commerce }\end{array}$ & Associé \\
\hline 12) & Kaufmann, Julius & $\begin{array}{l}\text { Grünbaum \& Kaufmann } \\
\text { Industrie et commerce textile, } \\
\text { puis Nationalzeitung } A G \\
\text { Presse et imprimerie }\end{array}$ & $\begin{array}{l}\text { Propriétaire (textile), } \\
\text { ensuite co-fondateur et } \\
\text { directeur commercial } \\
\text { (presse) }\end{array}$ \\
\hline & $\begin{array}{l}\text { Koenigs, Elise } \\
\text { et Felix }\end{array}$ & $\begin{array}{l}\text { Delbrück, Leo \& Co. } \\
\text { Banques et finances }\end{array}$ & Associé et directeur \\
\hline 14) & Liebermann, Max & $\begin{array}{l}\text { Peintre (son père et son grand } \\
\text { frère: industrie textile) }\end{array}$ & Indépendant \\
\hline
\end{tabular}

24. Ibid., p. 50. 


\begin{tabular}{|c|c|c|}
\hline 15) Loewe, Isidor & $\begin{array}{l}\text { Deutsche Waffen- und } \\
\text { Munitionsfabriken Ludwig } \\
\text { Loewe \& Co. (Usine d'armes et } \\
\text { munitions, avant : machines- } \\
\text { outils et machines à coudre) } \\
\text { Industrie et commerce }\end{array}$ & Propriétaire \\
\hline $\begin{array}{l}\text { 16) Mendelssohn, Franz } \\
\text { junior }\end{array}$ & $\begin{array}{l}\text { Mendelssohn \& Co. } \\
\text { Banques et finances }\end{array}$ & Copropriétaire, directeur \\
\hline 17) Mendelssohn, Robert & $\begin{array}{l}\text { Mendelssohn \& Co. } \\
\text { Banques et finances }\end{array}$ & Copropriétaire, directeur \\
\hline $\begin{array}{l}\text { 18) Mendelssohn- } \\
\text { Bartholdy, Ernst }\end{array}$ & $\begin{array}{l}\text { Mendelssohn \& Co. } \\
\text { Banques et finances }\end{array}$ & Copropriétaire, directeur \\
\hline 19) Merling, Paul & $\begin{array}{l}\text { Paul Merling } \\
\text { Système bancaire et finances }\end{array}$ & Propriétaire \\
\hline 20) Oppenheim, Hugo & $\begin{array}{l}\text { Robert Warschauer \& Co. } \\
\text { Banques et finances }\end{array}$ & Copropriétaire \\
\hline 21) Siemens, Georg & $\begin{array}{l}\text { Deutsche Bank } \\
\text { Banques et finances }\end{array}$ & Directeur \\
\hline 22) Simon, James & $\begin{array}{l}\text { Gebrüder Simon } \\
\text { Industrie textile / commerce }\end{array}$ & Copropriétaire \\
\hline 23) Staudt, Wilhelm & $\begin{array}{l}\text { Staudt und Comp. } \\
\text { Commerce et Banques }\end{array}$ & Employé, puis propriétaire \\
\hline 24) Steinbart, Karl & $\begin{array}{l}\text { Mendelssohn \& Co. } \\
\text { Banques et finances }\end{array}$ & Cadre \\
\hline 25) Steinthal, Max & $\begin{array}{l}\text { Deutsche Bank } \\
\text { Banques et finances }\end{array}$ & Directeur \\
\hline 26) Veit, Eduard & $\begin{array}{l}\text { Robert Warschauer \& Co. } \\
\text { Banques et finances }\end{array}$ & Copropriétaire \\
\hline $\begin{array}{l}\text { 27) Warschauer, Robert } \\
\text { (junior) }\end{array}$ & $\begin{array}{l}\text { Robert Warschauer \& Co. } \\
\text { Banques et finances }\end{array}$ & Copropriétaire \\
\hline 28) Wesendonk, Karl & $\begin{array}{l}\text { Friedrich-Wilhelms-Universität } \\
\text { Enseignement et sciences }\end{array}$ & Employé \\
\hline
\end{tabular}


Tableau 3 - La religion des mécènes et leurs ancêtres ${ }^{25}$

\begin{tabular}{|c|c|c|c|}
\hline $\begin{array}{l}\text { Nom, prénom } \\
\text { (année de naissance } \\
\text { et de décès) }\end{array}$ & $\begin{array}{l}\text { Ancêtres } \\
\text { juifs }\end{array}$ & $\begin{array}{l}\text { Appartenance } \\
\text { à la confession juive }\end{array}$ & $\begin{array}{l}\text { Abandon de } \\
\text { la confession } \\
\text { juive } \\
\text { (jusqu'en } \\
\text { 1910) }\end{array}$ \\
\hline $\begin{array}{l}\text { 1) Arnhold, Eduard } \\
(1849-1925)\end{array}$ & oui & oui & non \\
\hline $\begin{array}{l}\text { 2) Bernstein, Felicie } \\
(1852-1908)\end{array}$ & oui & oui & non \\
\hline $\begin{array}{l}\text { 3) Bleichröder, Georg } \\
(1857-1902)\end{array}$ & oui & oui & non \\
\hline $\begin{array}{l}\text { 4) } \begin{array}{l}\text { Bleichröder, Julius } \\
(1828-1907)\end{array}\end{array}$ & oui & oui & non \\
\hline $\begin{array}{l}\text { 5) Friedländer-Fuld, } \\
\text { Friedrich Viktor (Fritz) } \\
(1858-1917)\end{array}$ & oui & oui & $\begin{array}{l}\text { oui, } \\
\text { protestant, } \\
\text { baptisé le } \\
18 \text { décembre } \\
1896\end{array}$ \\
\hline $\begin{array}{l}\text { 6) Hagen (Levy), Carl } \\
(1856-1905)\end{array}$ & oui & oui & non \\
\hline $\begin{array}{l}\text { 7) Hainauer, née Prins, } \\
\text { Julie }(1850 \text { - 1926) }\end{array}$ & $\begin{array}{l}\text { Grandit dans } \\
\text { la famille } \\
\text { Reichenheim } \\
\text { (sans être pas } \\
\text { adoptée) }\end{array}$ & $\begin{array}{l}\text { Probablement. } \\
\text { Son mari Oscar Hainauer } \\
\text { était juif. }\end{array}$ & $\begin{array}{l}\text { Son mari né } \\
\text { en } 1840, \text { fut } \\
\text { baptisé le } 28 \\
\text { février } 1887 \\
\text { (protestant). }\end{array}$ \\
\hline $\begin{array}{l}\text { 8) Henckel von } \\
\text { Donnersmarck, } \\
\text { Guido }(1830-1916)\end{array}$ & non & non, protestant & -- \\
\hline $\begin{array}{l}\text { 9) von der Heydt, Karl } \\
(1858-1922)\end{array}$ & non & $\begin{array}{l}\text { non, protestant, son père } \\
\text { était calviniste. }\end{array}$ & -- \\
\hline $\begin{array}{l}\text { 10) Huldschinsky, Oscar } \\
(1846-1931)\end{array}$ & oui & oui & non \\
\hline $\begin{array}{l}\text { 11) Kappel, Marcus } \\
(1845-1919)\end{array}$ & oui & oui & non \\
\hline $\begin{array}{l}\text { 12) Kaufmann, Julius } \\
(1853-?)\end{array}$ & oui & oui & non \\
\hline $\begin{array}{l}\text { 13) Koenigs, Elise } \\
(1848-1932)\end{array}$ & non & non, catholiques & -- \\
\hline $\begin{array}{l}\text { 14) Liebermann, Max } \\
(1847-1935)\end{array}$ & oui & oui & non \\
\hline $\begin{array}{l}\text { 15) Loewe, Isidor } \\
(1848-1910)\end{array}$ & oui & oui & non \\
\hline
\end{tabular}

25. Ibid., p. 71. 


\begin{tabular}{|c|c|c|c|}
\hline $\begin{array}{l}\text { 16) Mendelssohn, } \\
\text { Franz junior } \\
(1865-1935)\end{array}$ & oui & $\begin{array}{l}\text { non, protestant, sa mère } \\
\text { sans ancêtres juifs, } \\
\text { son père de la famille } \\
\text { Mendelssohn et baptisé, } \\
\text { son grand-père Alexander } \\
\text { Mendelssohn juif et actif } \\
\text { dans la communauté juive, } \\
\text { mais il fit baptiser tous ses } \\
\text { enfants à leur naissance. }\end{array}$ & -- \\
\hline $\begin{array}{l}\text { 17) Mendelssohn, } \\
\text { Robert } \\
(1857-1917)\end{array}$ & oui & $\begin{array}{l}\text { non, protestant, voir son } \\
\text { frère }\left(n^{\circ} 16\right)\end{array}$ & -- \\
\hline $\begin{array}{l}\text { 18) Mendelssohn- } \\
\text { Bartholdy, Ernst } \\
(1846-1909)\end{array}$ & oui & $\begin{array}{l}\text { non, protestant, baptisé } \\
\text { le } 7 \text { février } 1847 \text { à Berlin, } \\
\text { son père baptisé avec ses } \\
\text { frères et sœurs le } 21 \text { mars } \\
1816 \text { à Berlin, ses grands- } \\
\text { parents paternels le } 4 \\
\text { octobre } 1822 \text { à Francfort- } \\
\text { sur-le-Main, les parents de } \\
\text { sa mère Albertine Heine } \\
\text { également baptisés, avec } \\
\text { des ancêtres juifs. }\end{array}$ & -- \\
\hline $\begin{array}{l}\text { 19) Merling, Paul } \\
(1853-1924)\end{array}$ & non & non, protestants & -- \\
\hline $\begin{array}{l}\text { 20) Oppenheim, Hugo } \\
(1847-1921)\end{array}$ & oui & $\begin{array}{l}\text { non, protestant, baptisé } \\
\text { le } 19 \text { mars } 1847, \text { voir } \\
\text { aussi son oncle }\left(n^{\circ} 27\right), \\
\text { ses cousins }\left(n^{\circ} 16 \text { et } n^{\circ}\right. \\
17) \text {, ses parents baptisés, } \\
\text { sa mère, membre de la } \\
\text { famille Mendelssohn, son } \\
\text { grand-père Alexander } \\
\text { Mendelssohn. }\end{array}$ & -- \\
\hline $\begin{array}{l}\text { 21) Siemens, Georg } \\
(1839-1901)\end{array}$ & non & non, protestants & -- \\
\hline $\begin{array}{l}\text { 22) Simon, James } \\
(1851-1932)\end{array}$ & oui & oui & non \\
\hline $\begin{array}{l}\text { 23) Staudt, Wilhelm } \\
(1852-1906)\end{array}$ & non & non, protestants & -- \\
\hline $\begin{array}{l}\text { 24) Steinbart, Karl } \\
(1852-1923)\end{array}$ & non (!) & non, protestants & -- \\
\hline $\begin{array}{l}\text { 25) Steinthal, Max } \\
(1850-1940)\end{array}$ & oui & oui & $\begin{array}{l}\text { Non, mais fit } \\
\text { baptiser ses } \\
\text { sept enfants. }\end{array}$ \\
\hline $\begin{array}{l}\text { 26) Veit, Eduard } \\
(1824-1901)\end{array}$ & oui & $\begin{array}{l}\text { non, protestant, son père } \\
\text { juif et baptisé en } 1843 \\
\text { après la mort de sa mère, } \\
\text { juive }\end{array}$ & -- \\
\hline $\begin{array}{l}\text { 27) Warschauer, Robert } \\
(1860-1918)\end{array}$ & oui & $\begin{array}{l}\text { non, protestant, } \\
\text { ses parents baptisés, } \\
\text { sa mère de la famille } \\
\text { Mendelssohn, } \\
\text { ses grands-parents } \\
\text { des deux côtés juifs }\end{array}$ & -- \\
\hline $\begin{array}{l}\text { 28) Wesendonk, Karl } \\
(1857-1934)\end{array}$ & non & non, protestants & -- \\
\hline
\end{tabular}


